\title{
$\widehat{A}$ Madridge
}

\section{madridge Journal of Internal and Emergency Medicine}

Interconnecting Scientific World

Open Access

\section{Public Health and Genomic Technologies: Microbiological Food Safety}

\author{
Andrea Ianni ${ }^{1 \star}$, Massimiliano Orsini ${ }^{2}$, Batistoni $C^{3}$, Roberto Virgili ${ }^{4}$, Michela Piredda ${ }^{5}$, Maria Grazia De Marinis ${ }^{5}$, \\ Giovanni Gherardi ${ }^{6}$, and Tommasangelo Petitti ${ }^{1}$ \\ 'Professor, Research Unit in Hygiene, Statistics and Public Health, Campus Bio-Medico University, Rome, Italy \\ ${ }^{2}$ Izsam Research Institute - Istituto Zooprofilattico Sperimentale dell'Abruzzo e del Molise “G. Caporale”, Teramo, Italy \\ ${ }^{3}$ Bachelor's Degree in Food Science and Human Nutrition, Campus Bio-Medico University, Rome, Italy \\ ${ }^{4}$ Anatomic and Surgical Pathology Service and Unit, Campus Bio-Medico University Hospital, Rome, Italy \\ ${ }^{5}$ Professor, Nursing Research Unit, Campus Bio-Medico University Medical School, Rome, Italy \\ ${ }^{6}$ Associate Professor, Medicine and Surgery Faculty, Campus Bio-Medico University Medical School, Rome, Italy
}

\section{Article Info}

*Corresponding author:
Andrea lanni
Professor
Research Unit in Hygiene, Statistics and
Public Health
Campus Bio-Medico University Hospital
Rome, Italy
E-mail: a.ianni@unicampus.it

Received: July 2, 2018

Accepted: July 5, 2018

Published: July 11, 2018

Citation: lanni A, Orsini M, Batistoni C, et al. Public Health and Genomic Technologies: Microbiological Food Safety. Madridge J Intern Emerg Med. 2018; 2(2): 67-70. doi: 10.18689/mjiem-1000114

Copyright: @ 2018 The Author(s). This work is licensed under a Creative Commons Attribution 4.0 International License, which permits unrestricted use, distribution, and reproduction in any medium, provided the original work is properly cited.

Published by Madridge Publishers

\begin{abstract}
Foodborne pathogens are major causes of morbidity and mortality throughout the world and the ability to conduct epidemiological investigations and identification studies in food borne illnesses represent a fundamental part of public health efforts. In the last decades, food borne pathogens have been increasingly studied due to their ability to change and adapt to different environmental and surviving conditions. Survival ability of those pathogens and the range of conditions for their growing has warranted the development and use of efficient techniques, for isolation, detection, differentiation, classification and typing. More recently, the development of novel and high-tech solutions for rapid, accurate, and non-laborious microbial detection methods has become imperative to improve the global food supply. Such solutions have begun to address the need for microbial detection that is faster and more sensitive than preexisting methodologies. Multiple technologies have been conventionally used in concert to develop rapid and accurate cell detection methods to qualify food products. Facilitating a wider range of downstream assays, molecular biology tools can increase microbial signals related to low initial numbers of target pathogen cells in food samples. Moreover, immunological methods have been combined with traditional laboratory methodologies to improve detection in food, as well the focus of recent pathogen efforts mostly on the advancement of biosensor technology, whose protocols utilize various nanotechnologies to produce biosensor platforms.
\end{abstract}

Among different technological solutions, whole genome sequencing (WGS) appears as essential for any full understanding of what microbes are doing and how they impact the environment around them, given that their mutation/selection efficiency contribute to their adaptability and survival chances. Moreover, the publicly available data resulting from these studies and the efforts of integrating them, exerted from different institutions, have multiple objectives. Data can be used to continue to build and support novel applications for food safety, as well as to potentiate surveillance and outbreak purposes. Integrated information have the power to answer to scientific questions pertaining to source attribution, antimicrobial resistance, transmission patterns, and virulence, which will further enable the protection and improvement of public health with respect to food borne disease.

In the present work - the introducing part of a deeper mini-review - it will be briefly discussed the methodology approach of combining traditional and molecular methods to differentiate and classify pathogens, in order to obtain efficient identification, 
outbreak investigation and epidemiological surveillance. Rapid and accurate detection of food borne pathogens can be regarded as essential for public health bio-surveillance to prevent foodborne infections and ensure the safety of foods.

\section{Abbreviations:}

WGS: Whole genome sequencing; WHO: World Health Organization; DALYs: Disability adjusted life years.

Keywords: Microbiological food safety; Genomics; molecular technologies; Foodborne pathogens; Public health.

\section{Introduction}

\section{Microbiological food safety}

Foodborne disease remains a substantial global challenge to public health. In the United States (U.S.) alone, according to the U.S. Food and Drug Administration, one in six or an estimated 48 million people have to face an infection or a disease due to foodborne pathogens (reported cases) annually, yelding 128,000 hospitalizations and 3,000 deaths [1]. As a relevant example of a developed country condition, the strict requirements of U.S. food chains and networks are regarded as among the safest in the world; however, one in six people suffer from contaminated foods or beverages in the U.S. each year $[1,2]$. In the WHO European Region, 23 million people fall ill and 5,000 people die every year for food contamination (biological, chemical and physical). In the European Region, 3 million people with less than 5 years fall ill from unsafe food annually: children less than 5 years old account for $13 \%$ of illness and $14 \%$ of deaths, almost representing $25 \%$ of total burden of disease [2]. Among cases of diarrheal foodborne disease in the European Region, with a total of more than 22 million illnesses per year, it has been estimated that 15 million cases are due to nor virus and close to 5 million due to campylobacteriosis [2,3]. Diarrheal disease accounts for $96 \%$ of foodborne illnesses and $62 \%$ of deaths. Among most severe cases (infectious lethal), which lead to almost 3,000 deaths per year in the European Region, almost 2,000 are due to non-typhoid Salmonella [3].

In this context, foodborne infection due to microbial contamination represents a growing health problem worldwide and over 200 different diseases are known to be transmitted by food [4]. Worldwide, it has been estimated that more than one billion of episodes of food poisoningrelated diarrhea occur annually and that billions of people in the world are at risk of unsafe foods [2,5]. Almost one in ten people in the world become ill every year due to consumption of contaminated food; diarrhoeal diseases are the most common cause of illness, with 550 million cases and 230,000 deaths every year [2]. Children under five years of age bear almost $40 \%$ of this burden, along with potentially life-long sequelae [1-4].

The burden of disease varies greatly across the world: WHO African and South-East Asia

Regions have the highest incidence and highest death rates (2). Public health burdens of disease can be measured in several ways, as by using disability adjusted life years (DALYs), quality adjusted life years (QALY) and cost-of-illness. Burden of disease evaluation can help policymakers to allocate appropriate resources for food safety control and intervention efforts [4]. The cited measures enable more comprehensive comparisons of infectious pathogens, with different patterns of incidence and outcome. In the European Region, the burden of foodborne diseases, as measured by DALYs per 100,000 among resident population, showed a range of 24-28 DALYs for diarrhoeal agents, 10-19 for invasive infectious disease agents and 0.4-6 for helminths [3].

Due to the globalization of the world's food trade, food has become a major pathway for human exposure to pathogenicmicrobials responsible for foodborne illness entering at many points along the value chain [5]. Thus, tracking and detecting pathogenic bacteria - and other microbes - in foods back to their sources can reveal crucial to obtain safety measures, fit for the producer, processor, distributor and consumer level.

In the present work, the first part of a mini-review on that theme, the contribution of molecular techniques for detecting and typing foodborne pathogens will be introduced, together with a brief view on molecular and genomic methods for food safety in public health.

\section{Advanced molecular technologies and food-borne pathogens detection}

Foodborne pathogens have represented a relevant threat to public health for a long time and are still the object of safety investigations. Surveillance studies have provided data and a better understanding into the existence and spread of foodborne pathogens [6]. The application of molecular techniques for detecting and typing of foodborne pathogens in surveillance has produced even more reliable epidemiological data for tracing the source of human infections [4]. Application of genomics tools, including comprehensive data analyses, has added important new insights into the biology and virulence of foodborne pathogens, allowing for precise definitions of foodborne pathogens on a subtype level [7]. Investigation on the spread of foodborne pathogen, and hence prevention of food safety hazard, has been increasingly extended on taxonomic details: beside that level, genomics-based data provide unique opportunities for improved species definitions, which may be useful for some pathogens where virulence characteristics are clearly linked to a particular species $[8,9]$.

A wide range of molecular techniques - including pulsed field gel electrophoresis, multilocus sequence typing, random amplified polymorphism deoxyribonucleic acid, repetitive extragenic palindromic, deoxyribonucleic acid sequencing, multiplex polymerase chain reaction and many more - have been used for detecting, speciating, typing, classifying and/or characterizing foodborne pathogens of great significance to humans $[9,10]$. Culture-based tests have been integrated with faster and sensitive culture independent diagnostic tools, including antigen-based assays and polymerase chain reaction (PCR) panels. The combination of techniques and the optimization of methods have increased the discriminatory 
power of detection and typing techniques applied [11]. In the last fourty years, molecular techniques have been increasingly and widely used in food safety surveillance, mutation and other genetic studies, to increase our understanding into the primary source of foodborne pathogens, source of infection and genetic diversity $[3,5,9]$.

The importance of genomic data in understanding foodborne pathogens and the integration of investigation techniques have further increased in recent years. The common genetic code is shared across all pathogens, regardless of the source of the isolates or sequenced technology used. This allows public health laboratories to build integrated networks, all utilizing the same raw data $[10,11]$. Moreover, innovative technology such as Nuclear Magnetic Resonance (NMR) coupled with nano particles can detect multiple target microbial pathogen DNA or proteins using nucleic acids, antibodies and other biomarkers assays analysis [7]. Finally, the development of a «multi-omics» approach has provided a new approach to the investigation of microbial communities allowing an integration of data, which can be used to better understand the behaviour of and interactions between community members. Omics represent valuable tools to produce behavioural and interactions data in microbial communities $[4,5,12]$. Approaches as the Next Generation Sequencing (NGS) produces data about ecology (metataxonomics), gene presence (metagenomics) and its transcription (metatranscriptomics). Metagenomic libraries can allow the reconstruction of pathogens genome then used for Genome Scale Metabolic Models (GSMM). Integrating omics data into Microbiological Risk Assessment (MRA) has an impact in food safety, but also to better control spoilage processes [12,13].

Metagenomics, metatranscriptomics, metaproteomics and metabolomics have the potential of producing a large amount of data in a very short time: a subsequent important effort consists in interpreting these data to assist risk management for food safety strategies and quality decisions [9]. Integrating multi-omics data in microbiological risk assessment can obtain such relevant results [12]. Among the different molecular tools developed, whole genome sequencing (WGS) has been applied to provide detailed characterization of foodborne pathogens [13]. Collecting the entire genome of a foodborne pathogen has been applied as a main characterization tool for investigating pathogenic characteristics. Because genomic data is inherited vertically, these data can be used to reconstruct the evolutionary history of food pathogens. Phylogenetics has emerged as a powerful tool used for many applications in foodborne outbreak detection and source tracking $[4,10,13]$.

Knowing which genes are present can represent the final step for better understanding of what these pathogens are doing through the expression of their RNAs. Knowing which proteins are being actively expressed allows deeper understanding of how pathogens survive and respond to their environments $[7,10]$.

Combination of omics tools, such as metatranscriptomics, can be useful to obtain a functional profile in a microbial community and reveal genetic interactions between foodborne pathogens and their food matrices. These results can be used efficiently to develop better prevention strategies, as well as to discover innovative methods for disinfection and biocontrol [12].

The classical model of intervention and safety strategy has been updated and adapted to this new scenario: the first step still remains prediction, followed by efforts and studies to understand microbial function/activities/regulatory mechanisms; the third (final) step is to translate knowledge and integrated information into effective intervention models and preventive controls.

\section{Methodological perspectives in public health}

To ensure food safety and to prevent unnecessary foodborne illnesses, rapid and accurate detection of pathogenic agents is essential. For the last two decades, culture-based tests have been supported by faster and sensitive culture-independent diagnostic methods, including antigen-based assays and polymerase chain reaction (PCR) panels [4]. Food is essential to life, hence food safety is regarded as a basic human right. Billons of people in the world are at risk of unsafe food [2]. Many millions become sick while hundreds of thousand die yearly. The WHO provides numbers of foodborne illnesses not only in terms of incidence but also number of deaths and DALYs as a measure of burden due to foodborne-related morbidity and mortality. All together, foodborne hazards have been recognized to cause an enormous human burden of 33 millions DALYs with $40 \%$ among children younger than 5 year-old [1-3].

The availability of traditional and genetic microbiological information about pathogens, which is collected and shared through public access, make it possible for each agency and research group to share the same information at the same time, in order to solve common public health problems [4], [5]. High throughput technologies and bioinformatics have improved public health control of food-borne infectious diseases by speeding outbreak detection and response, as well as improving preventive interventions and detecting emerging food-related risks [13]. International agencies have prompted several collaborating initiatives in order to accelerate and coordinate the integration of new technologies into practice. Collaboration and partnership among different national and international agencies and institutions are the key to strengthen efficacy of application for those methods and tools in public health [4]. Among the others, the WGS methodology highlights different roles that genomic sciences have gained in food safety and microbiological prevention. Food microbiology laboratories have changed as they integrate WGS-based tools into their traditional microbiology foundations [8,12]. This impact has been highlighted into the public health community with a rapid transitions and integration of genomic and metagenomic methods into foodborne disease surveillance and characterization programs. 


\section{Conclusion}

Molecular methodology has become crucial for any full understanding of what microbes are doing and how they impact the environments around them. This rapid characterization and linkage of pathogens to health consequences and risks will speed up investigations and ultimately improve food safety and public health.

\section{References}

1. Marder EP, Griffin PM, Cieslak PR, Dunn J, Hurd S, Jervis R, et al. Preliminary incidence and trends of infections with pathogens transmitted commonly through food-Foodborne Diseases Active Surveillance Network, 10 U.S. Sites, 2006-2017. MMWR Morb Mortal Wkly Rep. 2018; 67(11): 324-28. doi: 10.15585/mmwr.mm6615a1

2. World Health Organization (WHO).WHO estimates of the global burden of foodborne diseases: foodborne disease burden epidemiology reference group 2007-2015. WHO Library Cataloguing-in-Publication Data; 2015.

3. EFSA (European Food Safety Authority) and ECDC (European Centre for Disease Prevention and Control). The European Union summary report on trends and sources of zoonoses, zoonotic agents and food-borne outbreaks in 2016. EFSA J. 2017; 15(12): 5077 (228). doi. 10.2903/j.efsa.2017.5077

4. Gwinn M, MacCannell DR, Khabbaz RF. Integrating advanced molecular technologies into public health. J Clin Microbiol 2017; 55(3): 703-714. doi: 10.1128/JCM.01967-16

5. Fung F, Wang HS, Menon S. Food safety in the 21st century. Biomed J. 2018; 41(2): 88-95. doi: 10.1016/j.bj.2018.03.003
6. Mandal PK, Biswas AK, Choi K, Pal UK. Methods for rapid detection of foodborne pathogens: an overview. Am J Food Technol. 2011; 6(2): 87-102. doi: 10.3923/ajt.2011.87.102

7. Allard MW, Bell R, Ferreira CM, Gonzalez-Escalona N, Hoffmann M, Muruvanda $T$, et al. Genomics of foodborne pathogens for microbial food safety. Curr Opin Biotechnol. 2018; 49:224-29. doi: 10.1016/j.copbio.2017.11.002

8. Lukinmaa $\mathrm{S}$, Nakari UM, Eklund M, Siitonen A. Application of molecular genetics methods in diagnostics and epidemiology of food-borne bacterial pathogens. APMIS. 2004; 112(11-12): 908-929. doi: 10.1111/j.1600-0463.2004. apm11211-1213.x

9. Lambert D, Pightling A, Griffiths E, Van Domselaar G, Evans P, Berthelet S, et al. Baseline Practices for the Application of Genomic Data Supporting Regulatory Food Safety. J AOAC Int. 2017; 100(3): 721-731. doi: 10.5740/ jaoacint.16-0269

10. Ronholm J, Nasheri N, Petronella N, Pagotto F. Navigating microbiological food safety in the era of whole-genome sequencing. Clin Microbiol Rev. 2016; 29(4): 837-857. doi: 10.1128/CMR.00056-16

11. Adzitey F, Huda N, Ali GRR. Molecular techniques for detecting and typing of bacteria, advantages and application to foodborne pathogens isolated from ducks. 3Biotech. 2013; 3(2): 97-107. doi: 10.1007/s13205-012-0074-4

12. Cocolin $L$, Mataragas $M$, Bourdichon $F$, Doulgeraki A, Pilet M-F, Jagadeesan B, et al. Next generation microbiological risk assessment meta-omics: the next need for integration. Int J Food Microbiol. 2017; pii: S0168-1605(17)30502-0. doi: 10.1016/j.jfoodmicro.2017.11.008

13. Allard MW, Strain E, Melka D, Bunning K, Musser SM, Brown EW, et al. Timme R. Practical value of food pathogen traceability through building a whole-genome sequencing network and database. J Clin Microbiol. 2016; 54(8): 1975-1983. doi: 10.1128/JCM.00081-16 